\title{
Zerumbone alleviates chronic constriction injury-induced allodynia and hyperalgesia through serotonin 5-HT receptors
}

\begin{abstract}
Zerumbone, a bioactive sesquiterpene isolated from Zingiber zerumbet (Smith), has shown to exert antiallodynic and antihyperalgesic effects in neuropathic pain mice model in our recent study. The mechanism through which zerumbone alleviates neuropathic pain has yet to be elucidated. Thus, this study aimed to determine whether the serotonergic system, part of the descending pain modulation pathway, contributes to the antineuropathic effect of zerumbone. Participation of the serotonergic system in zerumbone-induced antiallodynia and antihyperalgesia was assessed using Dynamic Plantar Aesthesiometer von Frey test and Hargreaves plantar test respectively in chronic-constriction injury mice model. Administration of $\rho$-chlorophenylalanine (PCPA, $100 \mathrm{mg} / \mathrm{kg}$, i.p.) for four consecutive days to deplete serotonin (5-HT) prior to zerumbone administration blocked the antiallodynic and antihyperalgesic effects of zerumbone. Further investigation with 5-HT receptor antagonists methiothepin (5-HT 1/6/7 receptor antagonist, $0.1 \mathrm{mg} / \mathrm{kg}$ ), WAY-100635 (5-HT 1A receptor antagonist, $1 \mathrm{mg} / \mathrm{kg})$, isamoltane $(5-\mathrm{HT} 1 \mathrm{~B}$ receptor antagonist, $2.5 \mathrm{mg} / \mathrm{kg})$, ketanserin $(5-\mathrm{HT}$ $2 \mathrm{~A}$ receptor antagonist, $0.3 \mathrm{mg} / \mathrm{kg}$ ) and ondansetron (5-HT 3 receptor antagonist, $0.5 \mathrm{mg} / \mathrm{kg}$ ) managed to significantly attenuate antiallodynic and antihyperalgesic effects of zerumbone $(10 \mathrm{mg} / \mathrm{kg})$. These findings demonstrate that zerumbone alleviates mechanical allodynia and thermal hyperalgesia through the descending serotonergic system via 5 -HT receptors $1 \mathrm{~A}, 1 \mathrm{~B}$, 2A, 3, 6 and 7 in chronic constriction injury neuropathic pain mice.
\end{abstract}

Keyword: Zerumbone; Zingiber zerumbet; Neuropathic pain; Serotonin; Serotonin receptor 